Geometry $\&$ Topology

Volume 9 (2005) 315-339

Published: 23 February 2005

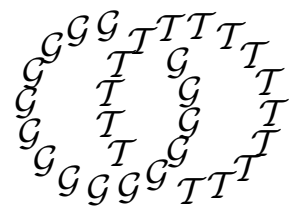

\title{
Periodic maps of composite order on positive definite 4 -manifolds
}

\author{
Allan L Edmonds \\ Department of Mathematics, Indiana University \\ Bloomington, IN 47405, USA \\ Email: edmonds@indiana.edu
}

\begin{abstract}
The possibilities for new or unusual kinds of topological, locally linear periodic maps of non-prime order on closed, simply connected 4-manifolds with positive definite intersection pairings are explored. On the one hand, certain permutation representations on homology are ruled out under appropriate hypotheses. On the other hand, an interesting homologically nontrivial, pseudofree, action of the cyclic group of order 25 on a connected sum of ten copies of the complex projective plane is constructed.
\end{abstract}

\section{AMS Classification numbers Primary: 57S17}

Secondary: $57 \mathrm{~S} 25,57 \mathrm{M} 60,57 \mathrm{~N} 14$

Keywords: Periodic map, 4-manifold, positive definite, permutation representation, pseudofree 


\section{Introduction}

The purpose of this paper is to explore the possibilities for unfamiliar kinds of topological, locally linear periodic maps of non-prime order on closed, simply connected 4-manifolds with positive definite intersection pairings.

Based on an earlier detailed study in Edmonds [6] of group actions on the $E_{8}$ 4-manifold, we conjectured that a locally linear group action on a closed, simply connected 4-manifold with positive definite intersection pairing induces a (perhaps signed) permutation representation on integral homology. For purely algebraic reasons these are exactly the kinds of representations that occur as automorphism groups of the standard pairing $n\langle+1\rangle$. We will in this paper, therefore, concentrate on the problem of what kinds of permutation representations can be realized, generally ignoring, however, the issue of nontrivially signed permutations, by restricting to odd periods. We will most often also concentrate on the prototypical case of $\#_{n} \mathbf{C} P^{2}$. We remark that the condition of being a permutation representation corresponds to the geometrical condition that the singular set for the group action contains no surfaces of positive genus. See [5], Proposition 2.4, for example.

It is well known and easy to see that every element of the permutation group $\Sigma_{n}$ acting on $n\langle+1\rangle$ can be realized by a homeomorphism (even a diffeomorphism) of $\#_{n} \mathbf{C} P^{2}$. Our question in this context becomes: Which elements of $\Sigma_{n}$ can be realized by a periodic homeomorphism? Of course, the answer to this question depends only on the conjugacy class of the permutation.

We will show that certain homology permutation representations do not arise. We also construct a new topological, locally linear, action of the cyclic group $C_{25}$ that has only a discrete singular set but is not semifree. It is possible, however, that all three conjectures hold as stated for smooth actions. See Section 4 for precise statements of theorems.

After an earlier version of this work was written M Tanase [16] and I Hambleton and Tanase [14 addressed some of the problems raised here in the smooth category. They showed using the equivariant Yang-Mills theory of Hambleton and $\mathrm{R}$ Lee [13] that certain permutation representations cannot be realized smoothly, including the action of $C_{25}$ mentioned above. They showed that any such smooth action has the same equivariant form and fixed point data of a standard equivariant connected sum of linear actions on copies of $\mathbf{C} P^{2}$. And they showed that an action that is smooth and pseudofree must be semifree, in contrast to the situation in the topological category that we display. 


\section{Notation and terminology}

We refer to [2] and [1] for generalities about transformation groups.

The conjugacy class of a permutation is determined by the associated partition of $n$ given by the cycles in the permutation. We might write $\left(p_{1}\right)\left(p_{2}\right) \ldots\left(p_{r}\right)$ where the $p_{i} \geq 1$ and $\sum p_{i}=n$. So, which conjugacy classes $\left(p_{1}\right)\left(p_{2}\right) \ldots\left(p_{r}\right)$ of $\Sigma_{n}$ can be realized by a periodic map $T$ of order $m=\operatorname{lcm}\left\{p_{1}, \ldots, p_{r}\right\}$ ?

Such an action of the cyclic group $C_{m}$ on the set $\{1,2, \ldots, n\}$ determines a permutation representation of $C_{m}$ on $\mathbf{Z}^{n}$. This representation we describe additively as $\left(p_{1}\right)+\left(p_{2}\right)+\cdots+\left(p_{r}\right)$

All group actions in this paper will be effective, so that the only group element that acts as the identity is the identity element. If a group $G$ acts on a space or just a set $X$ and $x \in X$, then the orbit of $x$ is $G(x)$. As a $G$-space $G(x) \approx G / H$ where $H=G_{x}$, the isotropy group of $x$. We refer to $G / H$, as the "orbit type", and to $H$ as the corresponding "isotropy type". When $G$ is cyclic, we can abbreviate subgroups and quotients to their integer orders. We will need to relate the various orbit types of an action to the conjugacy types of the corresponding representation on homology.

All group actions discussed in this paper will be locally linear, so that each point $x$ has a neighborhood invariant under the isotropy group $G_{x}$ on which the action of $G_{x}$ is equivalent to a linear action on a suitable euclidean space.

If $G$ acts locally linearly on a 4 -manifold $X$ with cyclic isotropy groups of odd order, then each singular point $x \in X$ has a local representation type, which can be described as a complex representation of the corresponding isotropy group. If $x$ is a fixed point of $G_{x} \approx C_{m}$, then this local representation can be described by the corresponding fixed point data, which is an ordered pair $(a, b)$, where a choice of generator $T \in G_{x}$ acts as $(z, w) \rightarrow\left(\zeta^{a} z, \zeta^{b} w\right)$, where $\zeta=\exp (2 \pi i / m)$, in local complex coordinates.

A group action is semifree if the only isotropy group that appears is the whole group, ie, if a nontrivial group element fixes a point, then every group element fixes that point. A cyclic group of prime order necessarily acts semifreely. A group action is said to be pseudofree if each nontrivial group element has a discrete fixed point set. Pseudofree actions are easier to study and amenable to geometric constructions and the application of surgery-based techniques, especially on 4-manifolds, since the fundamental group of the complement of the singular set is the same as the fundamental group of the ambient manifold. 


\section{Basic examples}

Here we record familiar basic linear actions of a finite cyclic group $C_{m}$ of order $m$ on projective spaces

Let $T$ be a periodic map of order $m$, which acts linearly on $S^{4} \subset \mathbf{C} \oplus \mathbf{C} \oplus \mathbf{R}$ by $T(x, y, t)=\left(\zeta^{a} x, \zeta^{b} y, t\right)$ or on $\mathbf{C} P^{2}$ by $T[x, y, z]=\left[\zeta^{a} x, \zeta^{b} y, z\right]$, where $\zeta=$ $\exp (2 \pi i / m)$. Now $\zeta^{a}$ has order $m / \operatorname{gcd}(a, m)$ and $\zeta^{b}$ has order $m / \operatorname{gcd}(b, m)$. To guarantee that $T$ actually has order $m$, and not less, we must require that $\operatorname{gcd}(a, b, m)=1$.

One obtains periodic maps on $S^{4}$ and $\mathbf{C} P^{2}$ with at most four orbit types (in the case of $S^{4}$ ) and at most five orbit types (in the case of $\mathbf{C} P^{2}$ ), depending on the choice of $m, a$, and $b$.

If $a \not \equiv b \bmod m$, then $T$ has exactly three fixed points: $[0,0,1],[0,1,0],[1,0,0]$ with local fixed point data $(a, b),(a-b,-b)$, and $(b-a,-a)$, respectively.

If $a \equiv b \bmod m$, then $T$ fixes the point $[0,0,1]$ and the 2 -sphere $[x, y, 0]$. The isolated point has local fixed point data $(a, a)$, the 2 -sphere has normal euler number +1 , and the generator $T$ rotates by $2 \pi a / m$ in the normal fiber.

Equivariant (anti-holomorphic) blow up of orbits yields similar actions with arbitrarily many singular points on $\#_{n} \mathbf{C} P^{2}$. One can write down the fixed point data for such a positive blow-up as follows. Start with the data

$$
(a, b),(a-b,-b),(b-a,-a) \text {. }
$$

Using the negative of the second entry we also have $\mathbf{C} P^{2}$ data

$$
(a-b, b),(a-2 b,-b),(2 b-a, b-a) \text {. }
$$

Equivariant connected sum yields an action on $\#_{2} \mathbf{C} P^{2}$ with data

$$
(a, b),(b-a,-a),(a-2 b,-b),(2 b-a, b-a) .
$$

One can then repeat the process. At each stage there are several options for the choice of point to blow up. Tanase [16] and Hambleton and Tanase [14 have given a thorough analysis of the possibilities for the fixed point data and the permutation representations that can arise when one iterates this construction.

\section{Statements of results}

We give three results here that show that under appropriate hypotheses certain permutation representations do not arise from topological, locally linear actions on a connected sum of copies of $\mathbf{C} P^{2}$. 
Theorem 4.1 For $p$ an odd prime, there is no locally linear action of $C_{m}$, $m=p^{k}, k \geq 3$, on $\#_{n} \mathbf{C} P^{2}$ (or any closed, simply connected, positive definite $4-$ manifold with $\left.b_{2}=n\right)$, where $n=p^{2}+p$ and the representation on homology is of type $(p)+\left(p^{2}\right)$.

Theorem 4.2 For $p$ an odd prime, there is no effective, locally linear, pseudofree, action of $C_{m}, m=p^{k}, k \geq 2$, on $\#_{n} \mathbf{C} P^{2}$ (or any closed, simply connected, positive definite 4-manifold) with homology representation of type

$$
\left(p^{\ell}\right)+r_{\ell+1}\left(p^{\ell+1}\right)+\cdots+r_{k}\left(p^{k}\right)
$$

where $1 \leq \ell \leq k-1$.

The proof involves the careful study of the $G$-Signature Formulas for $T$ and $T^{p}$, where $T$ denotes a chosen generator of $C_{m}$.

Theorem 4.3 There is no locally linear, pseudofree action of $C_{9}$ on $\#_{n} \mathbf{C} P^{2}$ (or any closed, simply connected, positive definite 4-manifold) with homology representation of type $r(9)+s(3)+t(1)$, where $t \leq 1$ and $s \geq 1$.

When $t=0$ and $s=1$, this is a consequence of Theorem 4.2, but it requires more work when $t=1$ or $s \geq 2$. The proof again involves a detailed look at the $G$-Signature Formulas for $T$ and $T^{3}$, where $T$ generates $C_{9}$. But the argument is more subtle.

In contrast to these non-existence results, we have one successful realization theorem.

Theorem 4.4 There is a locally linear pseudofree action of $C_{25}$ on $\#_{10} \mathbf{C} P^{2}$ with homology representation of type 2(5).

This result is especially interesting in light of the work of Tanase [16 and of Hambleton and Tanase [14 which shows that such an action cannot be smoothed.

Remark 4.5 From this result it is easy to use equivariant blow up to construct actions of $\mathrm{C}_{25}$ on $\#_{n} \mathbf{C} P^{2}$ with homology representation of type $r(25)+2(5)+$ $t(1)$, for any $r \geq 0$ and $t \geq 0$, where $n=10+25 r+t$.

In this case, suitable data that might come from an actual action is produced, in the sense that the $G$-Signature Formula would be satisfied. Then techniques developed in Edmonds and Ewing [8] are used to show that the data can actually be realized by a group action. One common thread of all these results is that in some sense the $G$-Signature Formula holds most of the key to the existence of group actions. 


\section{Tools}

Because they are crucial in what follows, we explicitly record two important and well-known tools for use in subsequent sections.

\subsection{Lefschetz Fixed Point Formula}

Let $T: X \rightarrow X$ generate an action of $C_{m}$ on $X$, a closed, simply connected 4-manifold. Then "local Smith theory" implies that $F=\operatorname{Fix}(T)$ consists of isolated points and surfaces. And the Lefschetz fixed point formula says that the Euler characteristic of the fixed point set is given by

$$
\chi(F)=\Lambda(T)=2+\operatorname{trace}\left[T_{*}: H_{2}(X) \rightarrow H_{2}(X)\right] .
$$

For a general discussion of this basic result see Allday and Puppe [1], (3.29). A refinement using a closer study of equivariant cohomology shows that all fixed surfaces are 2-spheres if and only if the representation on $\mathrm{H}_{2}$ is a permutation representation. See Edmonds [5], Proposition 2.4.

\section{$5.2 \quad G$-Signature Formula}

Let $T: X \rightarrow X$ generate an action of $C_{m}$ on a 4 -manifold $X$. In general, we understand the " $g$-signature" to be the character of a certain virtual complex representation evaluated on the generator $T$. One extends the intersection pairing on $H_{2}(X)$ to a Hermitian pairing on $H_{2}(X ; \mathbf{C})$ and forms the differences of the traces of $T_{*}$ on the positive and negative parts. But in the case of interest in this paper of positive definite 4-manifolds, it simplifies dramatically to

$$
\sigma(T, X):=\operatorname{trace}\left[T_{*}: H_{2}(X) \rightarrow H_{2}(X)\right]
$$

and assuming the representation on $H_{2}(X)$ is of permutation type, it is the number of fixed basis vectors in the permutation representation. In particular, it is a rational integer in this case.

Set $\zeta=\exp (2 \pi i / m)$. Suppose $T$ has isolated fixed points $x_{i}$ and fixed surfaces $S_{j}$. Suppose $T$ has local representation of "type" $\left(a_{i}, b_{i}\right)$ at $x_{i}$; let $S_{j}$ have normal euler number $n_{j}$ and normal rotation angle data $e_{j}$ (so that $T$ rotates an oriented normal plane to $S_{j}$ by $\left.2 \pi e_{j} / m\right)$ ). Then we have:

$$
\sigma(T, X)=\sum_{i} \frac{\left(\zeta^{a_{i}}+1\right)}{\left(\zeta^{a_{i}}-1\right)} \frac{\left(\zeta^{b_{i}}+1\right)}{\left(\zeta^{b_{i}}-1\right)}-\sum_{j} \frac{4 n_{j} \zeta^{e_{j}}}{\left(\zeta^{e_{j}}-1\right)^{2}}
$$

A nice reference for this version is Gordon [12]. 


\section{$6 \quad$ Number-theoretic issues}

This section may be skimmed or skipped and referred back to as needed. The issue is manipulating and drawing conclusions from the $G$-Signature Formula. The key results are Theorems 6.5 and 6.8 which are at least on the surface reminiscent of the Franz Independence Lemma [10.

We begin with a few very elementary but useful lemmas. If $\zeta=\exp (2 \pi i / m)$, then we can identify the cyclotomic field $\mathbf{Q}(\zeta)$ with $\mathbf{Q}[x] / \Phi_{m}(x)$, where $\Phi_{m}(x)$ is the $m$ th cyclotomic polynomial. Any elementary number theory book, for example [3], should be adequate reference.

Lemma 6.1 If $\zeta=\exp (2 \pi i / m)$, then $\zeta^{a}-1(a \not \equiv 0 \bmod m)$ has inverse in $\mathbf{Q}(\zeta)$ given by

$$
\left(\zeta^{a}-1\right)^{-1}=\frac{-1}{m} \prod_{\substack{i=1 \\(i \neq a)}}^{m-1}\left(1-\zeta^{i}\right) .
$$

Proof We have the complex polynomial factorization

$$
\left(x^{m}-1\right)=\prod_{i=0}^{m-1}\left(x-\zeta^{i}\right) .
$$

Dividing through by $x-1$ we have

$$
1+x+\cdots+x^{m-1}=\prod_{i=1}^{m-1}\left(x-\zeta^{i}\right) .
$$

Plug in $x=1$ to get

$$
m=\prod_{i=1}^{m-1}\left(1-\zeta^{i}\right)
$$

and isolate $\zeta^{a}-1=-\left(1-\zeta^{a}\right)$ to get the result.

Lemma 6.2 If

$$
\frac{\left(\zeta^{a}+1\right)}{\left(\zeta^{a}-1\right)}=\frac{\left(\zeta^{b}+1\right)}{\left(\zeta^{b}-1\right)}
$$

where $\zeta=\exp (2 \pi i / m)$, then $a \equiv b \bmod m$.

Cross-multiply and simplify to get $\zeta^{a}=\zeta^{b}$. The result follows. 
Lemma 6.3 If $\zeta=\exp (2 \pi i / m)$, then:

$$
\frac{\left(\zeta^{-a}+1\right)}{\left(\zeta^{-a}-1\right)}=-\frac{\left(\zeta^{a}+1\right)}{\left(\zeta^{a}-1\right)}
$$

Proof Multiply numerator and denominator on the left by $\zeta^{a}$ and simplify.

Lemma 6.4 The equation

$$
\frac{\left(\zeta^{a}+1\right)}{\left(\zeta^{a}-1\right)} \frac{\left(\zeta^{b}+1\right)}{\left(\zeta^{b}-1\right)}=1
$$

in which $\zeta=\exp (2 \pi i \mathrm{~m}), m$ odd, has no solutions $a$ and $b$.

Proof Clear denominators and simplify to show that $\zeta^{b}=-\zeta^{a}$. The result follows.

Theorem 6.5 Let $m=p^{t}$, where $p$ is an odd prime. If $\zeta=\exp (2 \pi i / m)$ and

$$
\frac{\left(\zeta^{a}+1\right)}{\left(\zeta^{a}-1\right)} \frac{\left(\zeta^{b}+1\right)}{\left(\zeta^{b}-1\right)}=\frac{\left(\zeta^{c}+1\right)}{\left(\zeta^{c}-1\right)} \frac{\left(\zeta^{d}+1\right)}{\left(\zeta^{d}-1\right)}
$$

in $\mathbf{Q}(\zeta)$, then $\{c, d\} \equiv \pm\{a, b\} \bmod m$.

Remark 6.6 It seems doubtful that Theorem 6.5 is true for any odd integer $m$. But the proof would definitely be more difficult in that case. Note that by applying a Galois automorphism of $\mathbf{Q}(\zeta) / \mathbf{Q}$, the hypothesis implies that the formula holds for $\zeta$ replaced by any primitive $m$ th root of unity. As a corollary, the formula holds for $\zeta$ replaced by any $m$ th root of unity at all.

Remark 6.7 Theorem 6.5 is equivalent to the statement that if

$$
\frac{\left(\zeta^{a}+1\right)}{\left(\zeta^{a}-1\right)} \frac{\left(\zeta^{b}+1\right)}{\left(\zeta^{b}-1\right)}+\frac{\left(\zeta^{c}+1\right)}{\left(\zeta^{c}-1\right)} \frac{\left(\zeta^{d}+1\right)}{\left(\zeta^{d}-1\right)}=0
$$

then $\{c, d\} \equiv \pm\{-a, b\} \bmod m$, which is the form in which we shall apply it.

Proof If $a= \pm c$ or $a= \pm d$ or $b= \pm c$ or $b= \pm d$, then the result follows from our analysis of simpler cases that arise when we divide both sides by common factors.

Cross-multiply and simplify to obtain:

$$
\zeta^{a+c+d}+\zeta^{b+c+d}+\zeta^{a}+\zeta^{b}=\zeta^{a+b+c}+\zeta^{a+b+d}+\zeta^{c}+\zeta^{d}
$$


It will suffice to show that some exponent on the left equals some exponent on the right $(\bmod m)$. To see this, focus, for example, on $a$. If it equals $a+b+c$ $\bmod m$, then $b \equiv-c$ and we are done, by the preceding observation. If it equals $a+b+d$, then $b \equiv-d$, and again we are done. If it equals $c$, then $a \equiv c$, and again we are done. And, finally, if it equals $d$, then $a \equiv d$, and again we are done. Similar considerations apply to any of the other exponents on the left. Altogether there would be 16 similar cases, which can be checked in ones head.

Now any element of $\mathbf{Q}(\zeta)$ has a unique expression as a polynomial in $\zeta$ of degree less than $\operatorname{deg}\left(\Phi_{m}(x)\right)$. In the special case when all the indicated exponents are less than $\operatorname{deg}\left(\Phi_{m}(x)\right)$, the result follows, since all the exponents on the left side must coincide with exponents on the right side.

The subsequent argument aims at showing that some exponent on the left must equal some exponent $\bmod p^{t}$ on the right in all cases.

Fix $m=p^{t}$. Let us say that an integer $k$ is ordinary $\bmod m$ if its minimal non-negative representative $\bar{k} \bmod m$ is less than $\operatorname{deg}\left(\Phi_{m}(x)\right)=p^{t}-p^{t-1}=$ $(p-1) p^{t-1}$. In this case the unique expression for $\zeta^{k}$ is simply $\zeta^{\bar{k}}$.

Similarly, let us say than an integer $k$ is critical $\bmod m$ if its minimal nonnegative representative $\bar{k}$ is greater than or equal to $\operatorname{deg}\left(\Phi_{m}(x)\right)$, ie, $(p-$ 1) $p^{t-1} \leq \bar{k} \leq p^{t}-1$ We must describe the unique representative of $\zeta^{k}$.

Recall that for $m=p^{t}$ the cyclotomic polynomial

$$
\Phi_{m}(x)=1+x^{p^{t-1}}+x^{2 p^{t-1}}+\cdots+x^{(p-1) p^{t-1}} .
$$

If $(p-1) p^{t-1} \leq \bar{k} \leq p^{t}-1$, then $\bar{k}=(p-1) p^{t-1}+\ell$, where, of course, $\ell=\bar{k}-(p-1) p^{t-1}$. Then the relation $x^{\ell} \Phi_{m}(x)=0$, holds in $\mathbf{Q}[x] / \Phi_{m}(x)$, that is

$$
x^{\ell}+x^{p^{t-1}+\ell}+x^{2 p^{t-1}+\ell}+\cdots+x^{(p-1) p^{t-1}+\ell}=0
$$

or

$$
x^{k}=-x^{k-p^{t}+p^{t-1}}-x^{k-p^{t}+2 p^{t-1}}-\cdots-x^{k-p^{t}+(p-1) p^{t-1}} .
$$

Thus when $k$ is critical mod $m$, the unique representative of $\zeta^{k}$ contributes $p-1$ terms, each with coefficient -1 . Note also that if $k_{1}$ and $k_{2}$ are both critical, but distinct $\bmod m$, then the unique representatives of $\zeta^{k_{1}}$ and $\zeta^{k_{2}}$ have no terms in common. This crucial observation is not at all clear in the case when $m$ is not a prime power.

Now let us return to our equation in the form

$$
\zeta^{a+c+d}+\zeta^{b+c+d}+\zeta^{a}+\zeta^{b}=\zeta^{a+b+c}+\zeta^{a+b+d}+\zeta^{c}+\zeta^{d}
$$


Since we already discussed the case when all exponents on the left side are ordinary, we can assume there is at least one critical exponent on the left hand side. This means that in the unique representation for the left hand side there is at least one negative term (e.g., there are actually two or three critical terms, or just one but $p-4 \geq 1$ ). Then the right hand side also has at least one negative term. But from the knowledge of the degree of a negative term we can determine the original degree (since $m$ is a prime power). Thus at least one exponent on the right hand side equals an exponent on the left hand side. Then we are done, as we observed at the beginning.

Strictly speaking we have to look more closely when $p=3$, where the left hand side has exactly one critical exponent and three ordinary terms exactly cancel off the $p-1=2$ negative terms from the representation of the critical term. But then it follows that the right hand side has exactly one positive ordinary term left, just as the left hand side must have. In particular there is an exponent that appears on both sides of the original equation, as needed.

Similar considerations are used to prove the following result.

Theorem 6.8 Let $m=p^{t}$, where $p$ is an odd prime. Suppose that $k \in \mathbf{Z}$ and that $c$ and $d$ are integers prime to $p$. Set $\zeta=\exp (2 \pi i / m)$. If

$$
\frac{-4 k \zeta}{(\zeta-1)^{2}}+p \frac{\left(\zeta^{c}+1\right)}{\left(\zeta^{c}-1\right)} \frac{\left(\zeta^{d}+1\right)}{\left(\zeta^{d}-1\right)}=p
$$

in $\mathbf{Q}(\zeta)$, then $k=p$, and $c \equiv d \equiv 1 \bmod p^{t}$ or $c \equiv d \equiv-1 \bmod p^{t}$.

Remark 6.9 When $k=p$, and $c \equiv d \equiv 1 \bmod p^{t}$, the formula is just $p$ times the $G$-Signature Formula for a standard periodic map on the complex projective plane. The theorem shows that there are no mysterious solutions to the $G$-signature equation in this case. It then follows that

$$
\frac{-4 k \xi}{(\xi-1)^{2}}+p \frac{\left(\xi^{c}+1\right)}{\left(\xi^{c}-1\right)} \frac{\left(\xi^{d}+1\right)}{\left(\xi^{d}-1\right)}=p
$$

for any $\xi=\zeta^{p^{j}}, j<t$.

Proof We may assume that $k>0$ by Lemma 6.4 By replacing $(c, d)$ by $(d, c)$ and/or $\left(p^{t}-c, p^{t}-d\right)$, we may assume that $1 \leq c \leq d \leq p^{t}-1$ and $c \leq \frac{p^{t}-1}{2}$.

Observe that $k$ must be divisible by $p$, as we can see by multiplying through by $\left(\zeta^{c}-1\right)\left(\zeta^{d}-1\right)$ and reducing modulo $(\zeta-1)$. Therefore one may write $k=p \ell$. Dividing through by $p$, then, we must solve the equation

$$
\frac{-4 \ell \zeta}{(\zeta-1)^{2}}+\frac{\left(\zeta^{c}+1\right)}{\left(\zeta^{c}-1\right)} \frac{\left(\zeta^{d}+1\right)}{\left(\zeta^{d}-1\right)}=1 .
$$


Clearing denominators, multiplying some things out, cancelling some terms and collecting and factoring, we obtain

$$
2 \ell \zeta\left(\zeta^{c}-1\right)\left(\zeta^{d}-1\right)=(\zeta-1)^{2}\left(\zeta^{c}+\zeta^{d}\right) .
$$

(As a quick check note that $c=d=\ell=1$ does satisfy this equation and moreover, if $c=d=1$, then it follows that $\ell=1$.)

Now multiply it all out and get:

$$
2 \ell \zeta^{c+d}+2 \ell=\zeta^{c+1}+\zeta^{d+1}+(2 \ell-2) \zeta^{c}+(2 \ell-2) \zeta^{d}+\zeta^{c-1}+\zeta^{d-1}
$$

When the left hand side and right hand side are each put into their unique form as a polynomial of degree less than $p^{t}-p^{t-1}$, we can compare constant terms. On the left hand side we either have $2 \ell \geq 2$ or 0 , and the latter only if $c+d \equiv-p^{t-1} \bmod p^{t}$.

Suppose $2 \ell \geq 2$ is the constant term on the left hand side. On the right hand side, then, the constant term can only have a positive contribution from a 0 power of $\zeta$. This could occur only from $c \equiv 1$ or $c \equiv-1$ or $d \equiv 1$ or $d \equiv-1$ $\left(\bmod p^{t}\right)$. This would limit us to $\ell=1$ and two of these four cases occuring. If $c \equiv d \equiv \pm 1 \bmod p^{t}$, we are done. The other possibility is $c \equiv 1 \bmod p^{t}$ and $d \equiv p^{t}-1 \bmod p^{t}$. But then $c+d$ becomes critical and the constant term on the left hand side would be 0 .

Thus we finally have to consider the case when the constant term on the left hand side is 0 , and somehow eliminate this possibility. That happens only when $c+d \equiv p^{t}-p^{t-1} \bmod p^{t}$. This clearly leads to a quick contradiction, but it remains to be seen how to organize the proof efficiently. In this situation the left hand side would have exactly $p-2$ terms in its reduced form:

$$
-2 \ell\left(\zeta^{p^{t-1}}+\zeta^{2 p^{t-1}}+\cdots+\zeta^{(p-2) p^{t-1}}\right)
$$

Since $c \geq 1$ and $c+d=p^{t}-p^{t-1}$, we see that

$$
d=p^{t}-p^{t-1}-c \leq p^{t}-p^{t-1}-1
$$

or

$$
d+1=p^{t}-p^{t-1}-c+1 \leq p^{t}-p^{t-1} .
$$

Thus the only possible critical exponent on the right hand side is $d+1$. And $d+1$ is critical only if

$$
d+1=p^{t}-p^{t-1}-c+1=p^{t}-p^{t-1}
$$


if and only if $c=1$. Thus, if there is a critical exponent on the right hand side, the right hand side can be rewritten as

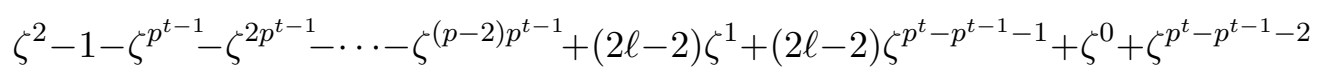

or

$(2 \ell-2) \zeta^{1}+\zeta^{2}-\zeta^{p^{t-1}}-\zeta^{2 p^{t-1}}-\cdots-\zeta^{(p-2) p^{t-1}}+(2 \ell-2) \zeta^{p^{t}-p^{t-1}-1}+\zeta^{p^{t}-p^{t-1}-2}$.

Thus on the right hand side the coefficient of $\zeta$ is $2 \ell-2$, while on the left hand side there is no $\zeta$ term unless $t=1$. But then the coefficient of $\zeta$ on the left hand side is $-2 \ell$. We conclude that $2 \ell-2=-2 \ell$, or $\ell=1 / 2$, a contradiction.

Finally we have to consider the case where no exponent on the right hand side is critical.

$$
-2 \ell\left(\zeta^{t-1}+\zeta^{2 p^{t-1}}+\cdots+\zeta^{(p-2) p^{t-1}}\right)=\zeta^{c+1}+\zeta^{d+1}+(2 \ell-2) \zeta^{c}+(2 \ell-2) \zeta^{d}+\zeta^{c-1}+\zeta^{d-1}
$$

In this case the right hand side has at least 3 distinct terms and at most 6 . But the left hand side has $p-2$ terms. Thus $3 \leq p-2 \leq 6$. Meanwhile on the left hand side all terms have the same coefficient of $-2 \ell$. The only way for some terms on the right hand side to coalesce would be to have $c=d$. (The other possibility of $c=d-1$ is ruled out because $c+d=p^{t}-p^{t-1}$ is even.) So our equation finally becomes

$$
-2 \ell\left(\zeta^{p^{t-1}}+\zeta^{2 p^{t-1}}+\cdots+\zeta^{(p-2) p^{t-1}}\right)=2 \zeta^{c+1}+(4 \ell-4) \zeta^{c}+2 \zeta^{c-1}
$$

where $c=\left(p^{t}-p^{t-1}\right) / 2$ and $p=5$. But then $-2 \ell=2$ and $-2 \ell=4 \ell-4$, a contradiction.

When manipulating the $G$-Signature Formula it once seemed natural to invoke the following "obvious fact".

Conjecture 6.10 $(m, p, t)$

If

$$
\sum_{i=1}^{t+2} \frac{\left(\zeta^{a_{i}}+1\right)}{\left(\zeta^{a_{i}}-1\right)} \frac{\left(\zeta^{b_{i}}+1\right)}{\left(\zeta^{b_{i}}-1\right)}=t
$$

where $\zeta=\exp (2 \pi i / m)$, and $a_{i}$ and $b_{i}$ are prime to $m$, then

$$
\sum_{i=1}^{t+2} \frac{\left(\zeta^{p a_{i}}+1\right)}{\left(\zeta^{p a_{i}}-1\right)} \frac{\left(\zeta^{p b_{i}}+1\right)}{\left(\zeta^{p b_{i}}-1\right)}=t
$$

where $p \mid m$. 
When $t=0$ this is equivalent to Theorem 6.5. The analogous statement is easily seen to be true for $p$ prime to $m$, because the two formulas are related by a Galois automorphism in that case. We will prove this for the smallest case not covered by earlier work: $p=3, m=9$ and $t=1$. But in general we will show that this natural conjecture is not true, for, say, $p=3, m=9$ and $t \geq 4$. (Perhaps it does hold for general $p$ when $t=1$, though.)

Remark 6.11 The special case above when $t=1$ corresponds to the situation of cyclic groups acting on $\mathbf{C} P^{2}$. In that case, under the assumption that the given equation holds for all $m$ th roots of unity, work of Edmonds and Ewing [7] shows that the only solutions come from standard linear actions.

Theorem 6.12 If

$$
\sum_{i=1}^{3} \frac{\left(\zeta^{a_{i}}+1\right)}{\left(\zeta^{a_{i}}-1\right)} \frac{\left(\zeta^{b_{i}}+1\right)}{\left(\zeta^{b_{i}}-1\right)}=1
$$

where $\zeta=\exp (2 \pi i / 9)$, and $a_{i}$ and $b_{i}$ are prime to 9 , then

$$
\sum_{i=1}^{3} \frac{\left(\zeta^{3 a_{i}}+1\right)}{\left(\zeta^{3 a_{i}}-1\right)} \frac{\left(\zeta^{3 b_{i}}+1\right)}{\left(\zeta^{3 b_{i}}-1\right)}=1
$$

Proof One can simply check this by brute force on a computer.

Example 6.13 There exist integers $(\bmod 9), a_{i}, b_{i}(i=1, \ldots, 8)$, such that

$$
\sum_{i=1}^{8} \frac{\left(\zeta^{a_{i}}+1\right)}{\left(\zeta^{a_{i}}-1\right)} \frac{\left(\zeta^{b_{i}}+1\right)}{\left(\zeta^{b_{i}}-1\right)}=6
$$

where $\zeta=\exp (2 \pi i / 9)$, and $a_{i}$ and $b_{i}$ are prime to 9 , but

$$
\sum_{i=1}^{8} \frac{\left(\zeta^{3 a_{i}}+1\right)}{\left(\zeta^{3 a_{i}}-1\right)} \frac{\left(\zeta^{3 b_{i}}+1\right)}{\left(\zeta^{3 b_{i}}-1\right)} \neq 6 .
$$

Proof This is not so easy to find by brute force. But one can reduce the problem to solving integral equations and eventually find examples. Of course, once one finds the examples, the verification that the examples work is essentially trivial.

Here is one solution: 


$$
\begin{aligned}
& 2 \frac{\left(\zeta^{1}+1\right)}{\left(\zeta^{1}-1\right)} \frac{\left(\zeta^{8}+1\right)}{\left(\zeta^{8}-1\right)}+3 \frac{\left(\zeta^{1}+1\right)}{\left(\zeta^{1}-1\right)} \frac{\left(\zeta^{2}+1\right)}{\left(\zeta^{2}-1\right)} \\
& \quad+\frac{\left(\zeta^{1}+1\right)}{\left(\zeta^{1}-1\right)} \frac{\left(\zeta^{4}+1\right)}{\left(\zeta^{4}-1\right)}+\frac{\left(\zeta^{2}+1\right)}{\left(\zeta^{2}-1\right)} \frac{\left(\zeta^{7}+1\right)}{\left(\zeta^{7}-1\right)}+\frac{\left(\zeta^{2}+1\right)}{\left(\zeta^{2}-1\right)} \frac{\left(\zeta^{4}+1\right)}{\left(\zeta^{4}-1\right)}=6
\end{aligned}
$$

But the same formula with $\zeta$ replaced by $\zeta^{3}$ yields 2 , not 6 .

\section{$7 \quad$ A non-existence result}

Here we give what in many ways is our best result, ruling out a particular homology representation without making any extra assumptions, such as the pseudofree hypothesis explored in the next section, about an action. In the smooth category the analog of this result follows from Hambleton and Tanase [14.

Theorem 7.1 (Restatement of Theorem 4.1) For $p$ an odd prime, there is no locally linear action of $C_{m}, m=p^{3}$, on $\#_{n} \mathbf{C} P^{2}$, where $n=p^{2}+p$ and the representation on homology is of type $(p)+\left(p^{2}\right)$.

Proof Suppose such an action exists. Let $T$ denote a generator of $C_{m}$. The homology representation implies that $\sigma(T, X)=0, \sigma\left(T^{p}, X\right)=p$ and $\sigma\left(T^{p^{2}}, X\right)=p^{2}+p$

We have inclusions

$$
\operatorname{Fix}(T) \subset \operatorname{Fix}\left(T^{p}\right) \subset \operatorname{Fix}\left(T^{p^{2}}\right)
$$

and $\operatorname{Fix}(T)=\operatorname{Fix}\left(T, \operatorname{Fix}\left(T^{p^{2}}\right)\right)$. Moreover, considering the Lefschetz Fixed Point formula, $\operatorname{Fix}(T)$ is two points or a 2 -sphere. Furthermore, in this case $\operatorname{Fix}(T)$ consists either of two fixed points (ie, $S^{0}$ ), with cancelling local fixed point data, or of a single 2-sphere $S^{2}$ with trivial normal bundle, as we see by applying the Lefschetz Fixed Point Formula and the $G$-Signature Formula to $T$.

Points of $\operatorname{Fix}\left(T^{p}\right)-\operatorname{Fix}(T)$ come in nontrivial orbits of size $p$. Let $\overline{\operatorname{Fix}(T)}$ denote the union of the components of $\operatorname{Fix}\left(T^{p}\right)$ meeting $\operatorname{Fix}(T)$, as it is a kind of closure. There are three cases:

(1) $\operatorname{Fix}(T)=S^{2}=\overline{\operatorname{Fix}(T)}$

(2) $\operatorname{Fix}(T)=S^{0}=\overline{\operatorname{Fix}(T)}$ 
(3) $\operatorname{Fix}(T)=S^{0} \subset \overline{\operatorname{Fix}(T)}=S^{2}$

We will argue that $\operatorname{Fix}(T)=S^{0}$ while $\overline{\operatorname{Fix}(T)}=S^{2}$ by eliminating the first two cases.

In any case we must have that

$$
\operatorname{Fix}\left(T^{p}\right)-\overline{\operatorname{Fix}(T)}=p \text { points. }
$$

For if

$$
\operatorname{Fix}\left(T^{p}\right)-\overline{\operatorname{Fix}(T)}=a S^{2}+b \text { points }
$$

then the Lefschetz formula implies that $2 a+b=p$. But $T$ acts on this set without fixed points. It follows that either $b=0$ or $b \geq p$. But if $b=0$, then $2 a=p$, contradicting the assumption that $p$ is odd. Thus $b \geq p$, from which it follows that $b=p$ and $a=0$. Similarly

$$
\operatorname{Fix}\left(T^{p^{2}}\right)-\operatorname{Fix}\left(T^{p}\right)=p^{2} \text { points. }
$$

Suppose now that Case 1 occurs, that is, $\operatorname{Fix}(T) \cong S^{2}$. Then, clearly, $\overline{\operatorname{Fix}(T)} \cong$ $S^{2}$ as well. Now

$$
\sigma(T, X)=0=\frac{-4 n_{j} \zeta^{e_{j}}}{\left(\zeta^{e_{j}}-1\right)^{2}}
$$

which forces the normal euler number to be 0 . The fixed point data at each of the $p$ new fixed points of $T^{p}$ must be the same, since $T$ permutes them transitively. Let it be $(e, f)\left(\bmod p^{2}\right)$. Then the $G$-Signature Formula for $T^{p}$ yields

$$
\sigma\left(T^{p}, X\right)=p=p \frac{\left(\zeta^{e}+1\right)}{\left(\zeta^{e}-1\right)} \frac{\left(\zeta^{f}+1\right)}{\left(\zeta^{f}-1\right)}
$$

which makes

$$
1=\frac{\left(\zeta^{e}+1\right)}{\left(\zeta^{e}-1\right)} \frac{\left(\zeta^{f}+1\right)}{\left(\zeta^{f}-1\right)}
$$

contradicting Lemma 6.4 We conclude that $\operatorname{Fix}(T) \cong S^{0}$.

Suppose next that $\operatorname{Fix}(T) \cong S^{0}$ and that $\overline{\operatorname{Fix}(T)} \cong S^{0}$ also. Let $(a, b)$ and $(c, d)$ be the local fixed point data for $T$ (and hence $T^{p}$, too) at the two points of $\operatorname{Fix}(T)$. Now the $G$-Signature Formula for $T$ says that

$$
\frac{\left(\zeta^{a}+1\right)}{\left(\zeta^{a}-1\right)} \frac{\left(\zeta^{b}+1\right)}{\left(\zeta^{b}-1\right)}+\frac{\left(\zeta^{c}+1\right)}{\left(\zeta^{c}-1\right)} \frac{\left(\zeta^{d}+1\right)}{\left(\zeta^{d}-1\right)}=0
$$

where $\zeta=\exp (2 \pi i / m)$. By Theorem 6.5, it follows that the fixed point data cancels and we may assume that $(c, d)=(a,-b)(\bmod m)$, for example. 
Again let the fixed point data at the $p$ new fixed points of $T^{p}$ be $(e, f)$. Then the $G$-Signature Formula for $T^{p}$ says that

$$
p \frac{\left(\xi^{e}+1\right)}{\left(\xi^{e}-1\right)} \frac{\left(\xi^{f}+1\right)}{\left(\xi^{f}-1\right)}=p
$$

where $\xi=\zeta^{p}=\exp \left(2 \pi i / p^{2}\right)$, which cannot hold, by Lemma 6.4 We therefore conclude that $\operatorname{Fix}(T)=S^{0}$ while $\overline{\operatorname{Fix}(T)}=S^{2}$.

Suppose that the 2-sphere $\overline{\operatorname{Fix}(T)}$ has normal euler number $k$. It then follows that the fixed point data for the two fixed points $(\operatorname{Fix}(T))$ must have the form $(a, b)$ and $(-a, b-k a)(\bmod m)$, as one sees by considering a standard model of the euler class $k$ bundle over $S^{2}$. Compare Lemma 9.3 below. On the other hand the $G$-Signature Formula for $T$ states that

$$
\frac{\left(\zeta^{a}+1\right)}{\left(\zeta^{a}-1\right)} \frac{\left(\zeta^{b}+1\right)}{\left(\zeta^{b}-1\right)}+\frac{\left(\zeta^{-a}+1\right)}{\left(\zeta^{-a}-1\right)} \frac{\left(\zeta^{b-k a}+1\right)}{\left(\zeta^{b-k a}-1\right)}=0
$$

where $\zeta=\exp (2 \pi i / m)$. By Theorem 6.5 the data cancels. Therefore we can conclude that $k \equiv 0 \bmod m$.

Then, as above, if the fixed point data at (each of) the $p$ new fixed points of $T^{p}$ is $(e, f)$. Then the $G$-Signature Formula for $T^{p}$ says that

$$
\sigma\left(T^{p}, X\right)=\frac{-4 k \xi^{b}}{\left(\xi^{b}-1\right)^{2}}+p \frac{\left(\xi^{e}+1\right)}{\left(\xi^{e}-1\right)} \frac{\left(\xi^{f}+1\right)}{\left(\xi^{f}-1\right)}=p
$$

where $\xi=\zeta^{p}=\exp \left(2 \pi i / p^{2}\right)$. This certainly has solutions, for example, when $k=p$ and $b=e=f$. According to Theorem 6.8 these are the only solutions, up to equivalence (replacing $(e, f)$ by $(-e,-f)$ or $(f, e))$.

Now consider $\operatorname{Fix}\left(T^{p^{2}}\right)$. This consists of $\operatorname{Fix}\left(T^{p}\right)$ plus $p^{2}$ points making up a single $T$ orbit and $p$ orbits of $T^{p}$. Let the $p^{2}$ new fixed points have local data $(c, d)$. Then the $\mathrm{g}$-Signature Formula for $T^{p^{2}}$ says that

$$
\sigma\left(T^{p^{2}}, X\right)=\frac{-4 k \mu^{b}}{\left(\mu^{b}-1\right)^{2}}+p \frac{\left(\mu^{b}+1\right)}{\left(\mu^{b}-1\right)} \frac{\left(\mu^{b}+1\right)}{\left(\mu^{b}-1\right)}+p^{2} \frac{\left(\mu^{c}+1\right)}{\left(\mu^{d}-1\right)} \frac{\left(\mu^{c}+1\right)}{\left(\mu^{d}-1\right)}=p+p^{2}
$$

where $\mu=\xi^{p}=\zeta^{p^{2}}=\exp (2 \pi i / p)$. It follows that

or

$$
p+p^{2} \frac{\left(\mu^{c}+1\right)}{\left(\mu^{d}-1\right)} \frac{\left(\mu^{c}+1\right)}{\left(\mu^{d}-1\right)}=p+p^{2}
$$

$$
\frac{\left(\mu^{c}+1\right)}{\left(\mu^{d}-1\right)} \frac{\left(\mu^{c}+1\right)}{\left(\mu^{d}-1\right)}=1
$$

contradicting Lemma 6.4 


\section{Pseudofree actions}

Here we investigate the possibilities for a pseudofree action that is not semifree. We first present a result that is analogous to the result that a prime (power) order map cannot have just one fixed point: a periodic map of odd prime power order cannot have just one singular orbit of smallest size.

Recent work of Hambleton and Tanase [14] shows that a pseudofree action must be semifree in the smooth category. So the actions considered here cannot be smoothed.

Theorem 8.1 (Restatement of Theorem4.2) For $p$ an odd prime, there is no effective, locally linear, pseudofree, action of $C_{m}, m=p^{k}, k \geq 2$, on $\#_{n} \mathbf{C} P^{2}$ (or any closed, simply connected, positive definite 4-manifold) with homology representation of type

$$
\left(p^{\ell}\right)+r_{\ell+1}\left(p^{\ell+1}\right)+\cdots+r_{k}\left(p^{k}\right)
$$

where $1 \leq \ell \leq k-1$.

Remark 8.2 An action with such a homology representation can exist for non-pseudofree actions. For example $C_{p^{2}}$ acts on $S^{4}$ with two fixed points and a 2 -sphere of points with isotropy group $C_{p}$. Blowing up one orbit of type $C_{p}$ and an arbitrary number of principal orbits creates the homology representation $(p)+r_{2}\left(p^{2}\right)$.

Proof Suppose such an action exists. Let $T$ denote a generator of $C_{m}$. The homology representation implies that $\sigma(T, X)=0$ and $\sigma\left(T^{p^{\ell}}, X\right)=p^{\ell}$.

We have inclusions

$$
\operatorname{Fix}(T)=\operatorname{Fix}\left(T^{p}\right)=\cdots=\operatorname{Fix}\left(T^{p^{\ell-1}}\right) \subset \operatorname{Fix}\left(T^{p^{\ell}}\right)
$$

and $\operatorname{Fix}(T)=\operatorname{Fix}\left(T, \operatorname{Fix}\left(T^{p^{\ell}}\right)\right)$. Moreover, $\operatorname{Fix}\left(T^{p^{i}}\right)$ is exactly two points for $i<\ell$, with cancelling local fixed point data, as we see by applying the Lefschetz Fixed Point Formula and the $G$-Signature Formula to $T^{p^{i}}$. Moreover, $\operatorname{Fix}\left(T^{p^{\ell}}\right)-\operatorname{Fix}(T)$ consists of a single orbit of $p^{\ell}$ points, as one can see from the Lefschetz formula.

Let $(a, b)$ and $(c, d)$ be the local fixed point data for $T$ (and hence $T^{p^{\ell}}$, too) at the two points of $\operatorname{Fix}(T)$. Now the $G$-Signature Formula for $T$ says that

$$
\frac{\left(\zeta^{a}+1\right)}{\left(\zeta^{a}-1\right)} \frac{\left(\zeta^{b}+1\right)}{\left(\zeta^{b}-1\right)}+\frac{\left(\zeta^{c}+1\right)}{\left(\zeta^{c}-1\right)} \frac{\left(\zeta^{d}+1\right)}{\left(\zeta^{d}-1\right)}=0
$$


where $\zeta=\exp (2 \pi i / m)$. By Theorem 6.5, it follows that the fixed point data cancels and we may assume that $(c, d)=(a,-b)$, for example.

Now let the fixed point data at the $p^{\ell}$ new fixed points of $T^{p^{\ell}}$ be $(e, f)$. Then the $G$-Signature Formula for $T^{p^{\ell}}$ says that

$$
p^{\ell} \frac{\left(\zeta^{e}+1\right)}{\left(\zeta^{e}-1\right)} \frac{\left(\zeta^{f}+1\right)}{\left(\zeta^{f}-1\right)}=p^{\ell}
$$

which cannot hold.

Theorem 8.3 (Restatement of Theorem 4.3) There is no locally linear, pseudofree action of $C_{9}$ on $\#_{n} \mathbf{C} P^{2}$ (or any closed, simply connected, positive definite 4-manifold) with homology representation of type

$$
r(9)+s(3)+t(1)
$$

where $t \leq 1$ and $s \geq 1$.

Proof We will begin by analyzing a general pseudofree action of $C_{m}$, where $m=p^{2}, p$ an odd prime. We will eventually get to a point where we can see that the cases covered by Theorem 4.3 (8.3) can be ruled out, but that the door remains open for $p \geq 5$.

So suppose that the cyclic group $C_{m}$ has generator $T$ and acts on $X=\#_{n} \mathbf{C} P^{2}$ with representation on $\mathrm{H}_{2}$ given by

$$
r(m)+s(p)+t(1)
$$

There are two kinds of singular orbits:

(1) $t+2$ fixed points $\left\{x_{i}\right\}$ with isotropy group $C_{m}=\langle T\rangle$. The fact that $\operatorname{card} X^{C_{m}}=t+2$ follows from the Lefschetz Fixed Point Formula.

(2) $s$ orbits of size $p$, with isotropy group $C_{p}=p C_{m}=\left\langle T^{p}\right\rangle$ :

$$
\left\{y_{i}, T y_{i}, \ldots, T^{p-1} y_{i}: i=1, \ldots, s\right\}
$$

Again, the fact that the number of such orbits is $s$ follows from the Lefschetz Fixed Point Formula.

We now interpret the $G$-Signature Formula in this situation. On the one hand we have

$$
\sigma(T, X)=t
$$

and

$$
\sigma\left(T^{p}, X\right)=t+p s .
$$


Now let $T$ have local representation of type $\left(a_{i}, b_{i}\right)$ at $x_{i}$. Set $\zeta=\exp (2 \pi i / m)$. Then we have:

$$
t=\sigma(T, X)=\sum_{i=1}^{t+2} \frac{\left(\zeta^{a_{i}}+1\right)}{\left(\zeta^{a_{i}}-1\right)} \frac{\left(\zeta^{b_{i}}+1\right)}{\left(\zeta^{b_{i}}-1\right)}
$$

There are plenty of solutions to this equation coming from equivariant connected sums of standard linear actions on $\mathbf{C} P^{2}$.

Similarly, let $T^{p}$ have local type $\left(c_{i}, d_{i}\right)\left(\bmod p\right.$ rotation numbers) at $y_{i}$. Then the type of $T^{p}$ at $T^{j} y_{i}$ is also $\left(c_{i}, d_{i}\right)$. In addition, the type of $x_{i}$ is again $\left(a_{i}, b_{i}\right)$, and the $G$-Signature Formula then yields

$$
t+p s=\sigma\left(T^{p}, X\right)=\sum_{i=1}^{t+2} \frac{\left(\xi^{a_{i}}+1\right)}{\left(\xi^{a_{i}}-1\right)} \frac{\left(\xi^{b_{i}}+1\right)}{\left(\xi^{b_{i}}-1\right)}+\sum_{i=1}^{s} p \frac{\left(\xi^{c_{i}}+1\right)}{\left(\xi^{c_{i}}-1\right)} \frac{\left(\xi^{d_{i}}+1\right)}{\left(\xi^{d_{i}}-1\right)}
$$

where $\xi=\exp (2 \pi i / p)=\zeta^{p}$. One would like to think that the two parts on each side of this equation correspond in the "obvious" way.

Under the substitution $\zeta \rightarrow \zeta^{p}$ we would obtain:

$$
t=\sigma(T, X)=\sum_{i=1}^{t+2} \frac{\left(\xi^{a_{i}}+1\right)}{\left(\xi^{a_{i}}-1\right)} \frac{\left(\xi^{b_{i}}+1\right)}{\left(\xi^{b_{i}}-1\right)}
$$

By Theorem 6.12 this holds for $p=3$ and $t \leq 1$, but does not hold in general. Whenever it does hold, however, we conclude that:

$$
s=\sum_{i=1}^{s} \frac{\left(\xi^{c_{i}}+1\right)}{\left(\xi^{c_{i}}-1\right)} \frac{\left(\xi^{d_{i}}+1\right)}{\left(\xi^{d_{i}}-1\right)}
$$

For $p=3$ this last equation has no solutions, since the terms in the sum are all $\pm \frac{1}{3}$, and we are done.

Even for $p>3$, if one can get to the last step of the proof, this formula does yield nontrivial restrictions. One cannot then have $s=1$, for example.

Remark 8.4 At first glance one might think that the equation

$$
s=\sum_{i=1}^{s} \frac{\left(\xi^{c_{i}}+1\right)}{\left(\xi^{c_{i}}-1\right)} \frac{\left(\xi^{d_{i}}+1\right)}{\left(\xi^{d_{i}}-1\right)}
$$

has no solutions. But in fact it does have solutions, at least for appropriate $p$ and $s$ : Build an equivariant connected sum of standard linear actions on $\#_{s} \mathbf{C} P^{2}$ (with $s+2$ fixed points) until one sees a canceling pair of fixed point data. Removing that data yields the desired algebraic solution. This procedure was 
used in earlier work of Edmonds and Ewing [8] to produce homologically trivial actions on a connected sum of copies of $\mathbf{C} P^{2}$ with nonstandard fixed point data not coming from an equivariant connected sum. Subsequently Hambleton and Lee 13 proved that some of these locally linear actions cannot be smoothable.

The smallest solution and the only one we know for $s \leq 2$ occurs for $p=5$ :

$$
\frac{\left(\zeta^{1}+1\right)}{\left(\zeta^{1}-1\right)} \frac{\left(\zeta^{4}+1\right)}{\left(\zeta^{4}-1\right)}+\frac{\left(\zeta^{2}+1\right)}{\left(\zeta^{2}-1\right)} \frac{\left(\zeta^{3}+1\right)}{\left(\zeta^{3}-1\right)}=2
$$

where $\zeta=\exp (2 \pi i / 5)$, which we will use in the next section.

We now turn our attention to the actual construction of an exotic, pseudofree, but not semifree, action of $C_{25}$, in the case when $t=0$ and $s=2$.

\section{An exotic $C_{25}$ action}

Here we give the details of the construction of a pseudofree, but not semifree, periodic map on a connected sum of copies of $\mathbf{C} P^{2}$.

Theorem 9.1 (Restatement of Theorem 4.4) There is a locally linear pseudofree action of $C_{25}$ on $\#_{10} \mathbf{C} P^{2}$ with homology representation of type 2(5).

Proof Based on the work in the preceding section we have an excellent picture of what such an action would have to look like. Let $T$ denote a generator of $C_{25}$. Then $T$ will have two fixed points, with canceling fixed point data. The fifth power $T^{5}$ will have two groups of five fixed points, in addition to the two original fixed points of $T$. Each of the five points in each group must have the same fixed point type, since the points are permuted cyclically by $T$.

We will use $(1,1),(1,24)$ for the fixed point data associated to the two fixed points of $T$. And we will have one orbit of five points all of which have $(1,4)$ as their local representation of $T^{5}(\bmod 5)$; and a second orbit of five points all of which have $(2,3)$ as their local representation of $T^{5}(\bmod 5)$. Thus, for the desired action on the expected 4-manifold we will have $\sigma\left(T, M^{4}\right)=0$ and $\sigma\left(T^{5}, M^{4}\right)=10$.

We will start with a 4-ball $D^{4}$ on which $T$ acts with local representation of type $(1,1)$. We will then add two orbits of five 2 -handles each along a carefully chosen equivariantly framed link. Each 2 -handle will be invariant under $\mathrm{T}^{5}$ and will contribute one isolated fixed point of $T^{5}$. The integral framings are 
determined mod 5 by the required local fixed point data. Our challenge is to find the appropriate 10 by 10 equivariant integral linking matrix with the additional requirement that the corresponding integral bilinear form is equivalent to the standard $10\langle+1\rangle$.

For reference we record three lemmas from Edmonds and Ewing [8]. For the lemmas suppose the generator $T$ of a free action of $C_{m}$ acts on $D^{4}=D^{4}(a, b)$ by $T(z, w)=\left(\zeta^{a} z, \zeta^{b} w\right)$, where $\zeta=\exp (2 \pi i / m)$ and $a$ and $b$ are prime to $m$.

Lemma 9.2 If $S_{x}$ and $S_{y}$ are two disjoint, invariant, simple closed curves in $\partial D^{4}(a, b)$ on which the generator $T$ of $C_{m}$ operates by rotation by $2 \pi x / m$ and $2 \pi y / m$, respectively, then $L k\left(S_{x}, S_{y}\right) \equiv a b x^{\prime} y^{\prime} \bmod m$, where $x x^{\prime} \equiv 1$ $\bmod m$ and $y y^{\prime} \equiv 1 \bmod m$.

Recall the standard observation that in the orbit space $\partial D^{4} / C_{m}$ the simple closed curve $\bar{S}_{x}$ represents $T^{x^{\prime}}$ of $\pi_{1}\left(\partial D^{4} / C_{m}\right)$ under the standard identification with $C_{m}=\langle T\rangle$, where $x x^{\prime} \equiv 1 \bmod m$.

Lemma 9.3 Let $f: S^{1} \times D^{2} \rightarrow S^{1} \times D^{2}$ by $f(z, w)=\left(z, z^{r} w\right)$. If $C_{m}$ acts on the target $S^{1} \times D^{2}$ by $T(z, w)=\left(\zeta^{a} z, \zeta^{b} w\right)$, then $f$ is equivariant with respect to the action on the domain given by $T(z, w)=\left(\zeta^{a} z, \zeta^{b-r a} w\right)$.

Lemma 9.4 Suppose a 2-handle is added to $D^{4}(a, b)$ along a curve $S_{k} \subset$ $\partial D^{4}(a, b)$, with framing $r$. Then the framing map can be chosen so that the action of $C_{m}$ extends over the 2-handle $B^{2} \times D^{2}$ with action of type $(-k,-r k+$ $\left.a b k^{\prime}\right) \bmod m$.

Now back to the situation of $m=25$ and of $C_{25}$ acting on $\mathrm{D}^{4}(1,1)$. To add a 2-handle invariant under $T^{5}$ that gives rise to a fixed point of $T^{5}$ of type $(1,4)$ we need a curve $S_{-1}=S_{4}$ on which $T^{5}$ acts by rotation by $-2 \pi / 5$ and with framing $r$ so that $-r *(-1)+1 * 1 *(-1) \equiv 4 \bmod 5$, and hence $r \equiv 0 \bmod 5$. We will use five such curves, cyclically permuted by $T$.

Similarly, to add a 2-handle invariant under $T^{5}$ that gives rise to a fixed point of $T^{5}$ of type $(2,3)$ we need a curve $S_{-2}=S_{3}$ on which $T^{5}$ acts by rotation by $-2 * 2 \pi / 5$ and with framing $r$ so that $-r *(-2)+1 * 1 * 2 \equiv 3 \bmod 5$, and hence $r \equiv 3 \bmod 5$. We will also use five such curves, again cyclically permuted by $T$.

We obtain these curves by representing $T^{20}$ and $T^{10}$, respectively, by disjoint simple closed curves in $L=\partial D^{4}(1,1) / C_{25}$ using the canonical identification of $\pi_{1}(L)$ with $C_{25}$. 
Attaching two 2-handles downstairs, or ten 2-handles upstairs, we end up with a 2 by 2 linking matrix over $\mathbf{Z}\left[C_{25}\right]$. If $W$ is the resulting 4 -manifold, then $H_{2}\left(W ; \mathbf{Z}\left[C_{25}\right]\right)=H_{2}(\widetilde{W} ; \mathbf{Z})=\mathbf{Z}\left[C_{5}\right] \oplus \mathbf{Z}\left[C_{5}\right]$. The $\mathbf{Z}\left[C_{25}\right]$-valued linking form depends on the exact choice of curves and integral framings and integral linking numbers. But it is determined mod 5 by the above lemmas as:

$$
\left[\begin{array}{cc}
5+T^{5}+T^{10}+T^{15}+T^{20} & 3+3 T^{5}+3 T^{10}+3 T^{15}+3 T^{20} \\
3+3 T^{5}+3 T^{10}+3 T^{15}+3 T^{20} & 3+4 T^{5}+4 T^{10}+4 T^{15}+4 T^{20}
\end{array}\right]
$$

Moreover, having made one tentative choice of these curves and their framings, one can make some changes by sliding a small arc on one curve across another of the curves and extending equivariantly. The upshot of this is that one can achieve any integral matrix of the form

$$
\left[\begin{array}{cc}
a_{0}+a_{1}\left(T^{5}+T^{20}\right)+a_{2}\left(T^{15}+T^{10}\right) & b_{0}+b_{1}\left(T^{5}+T^{20}\right)+b_{2}\left(T^{15}+T^{10}\right) \\
b_{0}+b_{1}\left(T^{5}+T^{20}\right)+b_{2}\left(T^{15}+T^{10}\right) & c_{0}+c_{1}\left(T^{5}+T^{20}\right)+c_{2}\left(T^{15}+T^{10}\right)
\end{array}\right]
$$

where $a_{0} \equiv 0 \bmod 5, a_{1} \equiv a_{2} \equiv 4 \bmod 5, b_{0} \equiv b_{1} \equiv b_{2} \equiv 3 \bmod 5, c_{0} \equiv 3$ $\bmod 5, c_{1} \equiv c_{2} \equiv 4 \bmod 5$. The challenge is to choose the integers $a_{0}, \ldots, c_{2}$ so that the resulting underlying rank 10 integral form is unimodular and in fact represents $10\langle+1\rangle$.

It took some time to find such a matrix, by a combination of brute force computer searching and judicious simplifications. Here is one such matrix:

$$
\left[\begin{array}{cc}
45+16\left(T^{5}+T^{20}\right)+16\left(T^{15}+T^{10}\right) & 93+23\left(T^{5}+T^{20}\right)+23\left(T^{15}+T^{10}\right) \\
93+23\left(T^{5}+T^{20}\right)+23\left(T^{15}+T^{10}\right) & 198+29\left(T^{5}+T^{20}\right)+29\left(T^{15}+T^{10}\right)
\end{array}\right]
$$

One can check that this matrix has determinant 1 in $\mathbf{Z}\left[C_{25}\right]$ !

The corresponding integer-valued form is given by:

$\left[\begin{array}{cccccccccc}45 & 16 & 16 & 16 & 16 & 93 & 23 & 23 & 23 & 23 \\ 16 & 45 & 16 & 16 & 16 & 23 & 93 & 23 & 23 & 23 \\ 16 & 16 & 45 & 16 & 16 & 23 & 23 & 93 & 23 & 23 \\ 16 & 16 & 16 & 45 & 16 & 23 & 23 & 23 & 93 & 23 \\ 16 & 16 & 16 & 16 & 45 & 23 & 23 & 23 & 23 & 93 \\ 93 & 23 & 23 & 23 & 23 & 198 & 29 & 29 & 29 & 29 \\ 23 & 93 & 23 & 23 & 23 & 29 & 198 & 29 & 29 & 29 \\ 23 & 23 & 93 & 23 & 23 & 29 & 29 & 198 & 29 & 29 \\ 23 & 23 & 23 & 93 & 23 & 29 & 29 & 29 & 198 & 29 \\ 23 & 23 & 23 & 23 & 93 & 29 & 29 & 29 & 29 & 198\end{array}\right]$

One can check directly that this matrix has determinant 1 in $\mathbf{Z}$, is positive definite, and in fact can be reduced to a $10 \times 10$ identity matrix by simultaneous integral row and column operations. Therefore it represents the standard 
intersection form $10\langle+1\rangle$ (as opposed to $E_{8} \oplus 2\langle+1\rangle$ ). It helps to use a computer algebra package, not only to find the matrix, but even just to verify its claimed properties. Alternatively one might be able to take a more theoretical approach, showing that there exist three independent vectors of norm 1 and invoking the known classification of unimodular forms of low rank. Or one might be able to apply N. Elkies's [9] characterization of the standard lattice by showing that the present lattice has no "short characteristic vectors". We have, however, opted for a more direct and naïve approach. See the end of this section for the Maple code used to make these direct verifications.

Form a smooth 4 -manifold $W^{4}$ with smooth $C_{25}$ action by attaching these ten 2 -handles to $D^{4}(1,1)$ equivariantly. The matrix above gives the intersection pairing on $\mathrm{H}_{2}(W)$. Because the intersection pairing is unimodular, the boundary $\Sigma$ of $W^{4}$ is an integral homology 3-sphere. According to Freedman's fundamental work [11], such a homology 3 -sphere is the boundary of a compact contractible, topological 4 -manifold $\Delta$. We must extend the action on $\Sigma$ to a locally linear action on $\Delta$. This problem was completely analyzed in Edmonds [4] for $m$ prime, and then in Kwasik and Lawson [15] for general $m$. The necessary and sufficient conditions are that the quotient $Q=\Sigma / C_{m}$ have the same signature invariants ( $\rho$ or $\alpha$ invariants) as some lens space, and the same Reidemeister torsion, up to squares, of the same lens space.

Our choice of fixed point data guarantees that the signature invariants are correct.

The torsion condition comes down to the issue of the determinant of the above matrix over $\mathbf{Z}\left[C_{25}\right]$ being a square of a unit in $\mathbf{Z}\left[C_{25}\right] /(N)$, where $N \in \mathbf{Z}\left[C_{25}\right]$ denotes the sum of the group elements. See the argument in Edmonds and Ewing [8], pages 1115-1117. We have the good fortune that this determinant is in fact 1 in $\mathbf{Z}\left[C_{25}\right]$.

Remark 9.5 What about the prospects of similar actions for $C_{m}, m=p^{2}$, $p>5$ ? We can find suitable fixed point data, although the number of terms necessary seems to grow with $p$. The problem of lifting the data to find a suitable positive definite linking matrix, however, seems formidable at the present time. In addition it is unclear whether the torsion issue would be so easily resolvable in general.

\section{Maple Code for Matrix Reduction}

The following Maple code shows that the $10 \times 10$ matrix discussed above can be reduced over the integers to the identity matrix by a suitable sequence of 78 simultaneous row and column operations. 


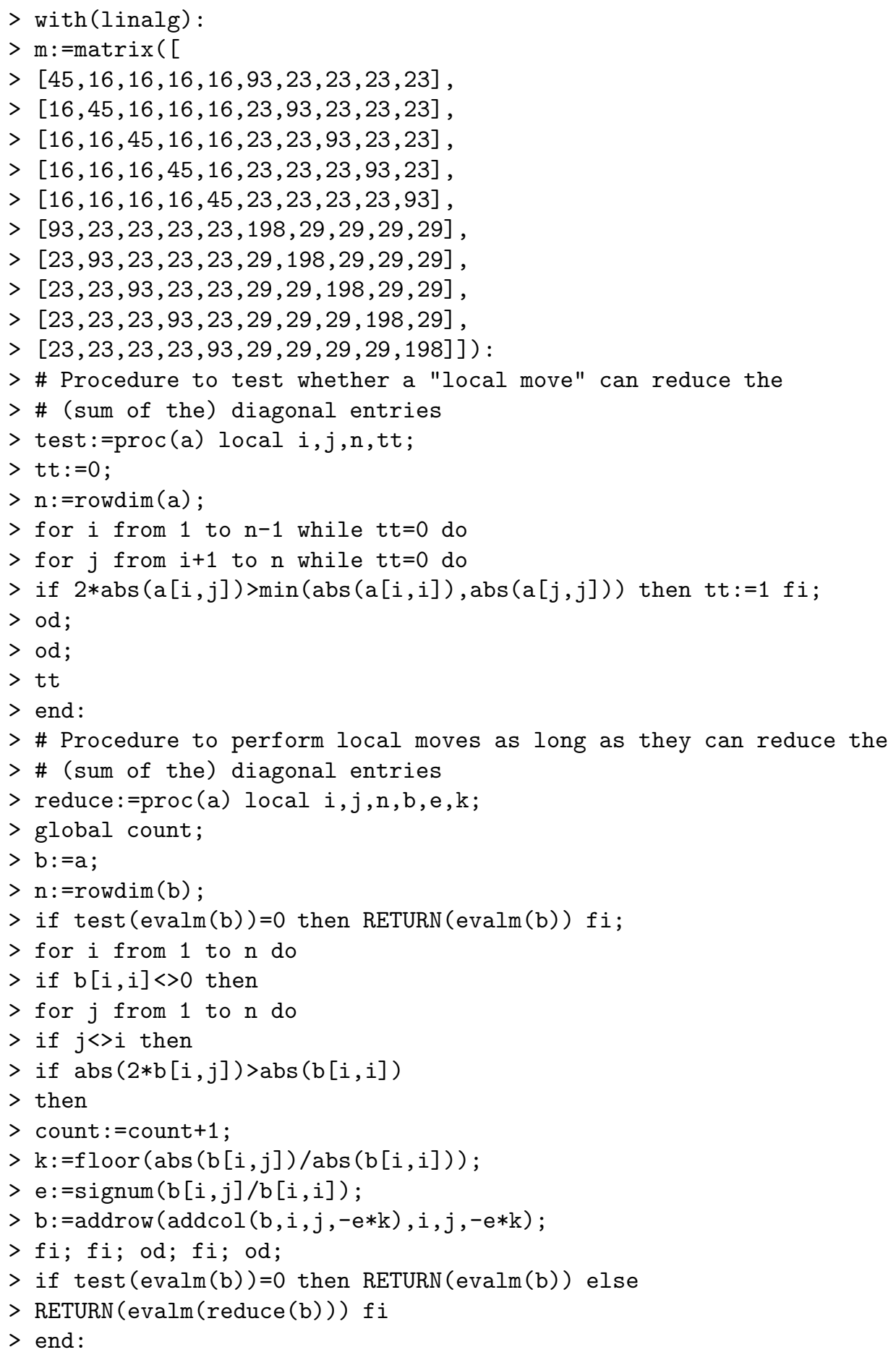




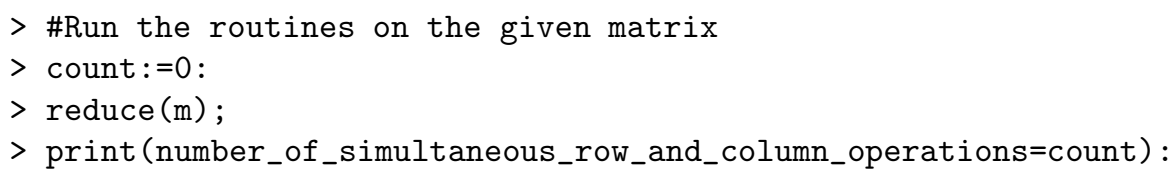

\section{References}

[1] C Allday, V Puppe, Cohomological Methods in Transformations Groups, Cambridge studies in Advanced Mathematics 32, Cambridge Univ. Press (1993) MathReview

[2] G E Bredon, Introduction to Compact Transformation Groups, Pure and Applied Mathematics 46, Academic Press (1972) MathReview

[3] Z I Borevich, I R Shafarevich, Number Theory, Academic Press (1966) MathReview

[4] A L Edmonds, Construction of group actions on four-manifolds, Trans. Amer. Math. Soc. 299 (1987) 155-177 MathReview

[5] A L Edmonds, Aspects of group actions on four-manifolds, Topology Appl. 31 (1989) no. 2, 109-124. MathReview

[6] A L Edmonds, Automorphisms of the $E_{8}$ four-manifold, from: "Geometric topology (Athens, GA, 1993)", AMS/IP Stud. Adv. Math. 2.1, Amer. Math. Soc. Providence, RI (1997) 282-299 MathReview

[7] A L Edmonds, J H Ewing, Locally linear group actions on the complex projective plane, Topology 28 (1989) 211-223 MathReview

[8] A L Edmonds, J H Ewing, Realizing forms and fixed point data in dimension four, Amer. J. Math. 114 (1992) 1103-1126 MathReview

[9] N D Elkies, A characterization of the $Z^{n}$ lattice, Math. Res. Lett. 2 (1995) 321-326 MathReview

[10] W Franz, Über die Torsion einer Überdeckung, J. Reine Angew. Math. 173 (1935) 245-254

[11] M H Freedman, The topology of four-dimensional manifolds, J. Differential Geom. 17 (1982) 357-453 MathReview

[12] C Gordon, On the $G$-signature theorem in dimension four, from: "À la Recherche de la Topologie Perdue", (L Guillou and A Marin, editors) Progr. Math. 62, Birkhäuser (1986) 159-180 MathReview

[13] I Hambleton, R Lee, Smooth group actions on definite 4-manifolds and moduli spaces, Duke Math. J. 78 (1995) 715-732 MathReview

[14] I Hambleton, M Tanase, Permutations, isotropy and smooth cyclic group actions on definite 4-manifolds, Geom. Topol. 8 (2004) 475-509 MathReview

[15] S Kwasik, T Lawson, Nonsmoothable $Z_{p}$ actions on contractible 4 -manifolds, J. Reine Angew. Math. 437 (1993) 29-54 MathReview

[16] M Tanase, Smooth finite cyclic group actions on positive definite 4-manifolds, PhD Thesis, McMaster University (2003) 